\title{
Microsatellite marker development based on next-generation sequencing for the smooth marron (Cherax cainii, Austin) and cross-species amplification in other Cherax species
}

\author{
Shannon R. Loughnan ${ }^{1 *}$, Luciano B. Beheregaray ${ }^{1}$ and Nicholas A. Robinson ${ }^{1,2}$
}

\begin{abstract}
Background: The smooth marron, Cherax cainii is an important freshwater crustacean species for aquaculture and for a local wild fishery. C. tenuimanus, commonly known as the hairy marron is under threat from environmental impacts and genetic introgression from C. cainii that is hampering the survival of wild C. tenuimanus stocks. Marron are endemic to the south-west of Western Australia and C. tenuimanus is restricted to only the Margaret River.

Results: To isolate microsatellite sequences, shotgun 454 pyrosequencing was performed resulting in 184,981 DNA sequence reads. Following screening for microsatellites, 8799 putative microsatellite loci were detected and PCR primers were designed for 968 of these. Ten microsatellite loci were screened in 30 captive C. cainii individuals with eight loci producing unambiguous results. The average number of alleles per locus was 4.7 and average $H_{e}$ was 0.474 . Following an analysis of relatedness, $79 \%$ of captive dyads were assigned as unrelated. Utilising C. quadricarinatus and C. destructor, cross-species amplification tests were conducted and amplification was achieved at four of the eight loci.

Conclusions: Using next-generation sequencing methods, eight polymorphic microsatellite loci were developed from C. cainii, with potential for cross amplification in other Cherax species. The markers can be utilised for studies of natural genetic stock structure and for monitoring relatedness levels and genetic variation in both wild and captive populations.
\end{abstract}

Keywords: Marron, Cherax cainii, Cherax tenuimanus, Microsatellites, Next-generation sequencing

\section{Findings}

Commonly known as the smooth marron, Cherax cainii (Austin) is a riverine crustacean species endemic to the south-west of Western Australia, which is managed as a wild fishery but also has high aquaculture potential with many commercial facilities under operation. Future studies of C. cainii will benefit from the development of polymorphic microsatellite markers from next-generation

\footnotetext{
*Correspondence: shannon.loughnan@flinders.edu.au

1 Molecular Ecology Laboratory, School of Biological Sciences, Flinders

University, PO Box 2100, Adelaide 5001, SA, Australia

Full list of author information is available at the end of the article
}

sequencing (NGS), which can be employed as a tool for the analysis of wild genetic stock structure, captive genetic diversity and genetic improvement (for determining broodstock pedigrees, genetic diversity and inbreeding levels). Polymorphic genetic markers can also be potentially utilised in the conservation of wild C. tenuimanus (Smith) stocks, a species commonly known as the hairy marron. This species has become critically endangered mainly due to the introduction of $C$. cainii into the restricted habitat of $C$. tenuimanus [1], and there are concerns that interbreeding and outbreeding depression may cause the extinction of C. tenuimanus. To date, 13 microsatellite markers have been developed and used to 
explore the genetic introgression of $C$. cainii and C. tenuimanus [2]. The microsatellite loci developed in the current study provide an important addition to the suite of markers for this genus, especially to improve analyses of parentage and relatedness in captive populations.

Total genomic DNA was extracted using a modified salting out procedure [3] for NGS, from a piece of tissue sourced from the abdomen of one $C$. cainii stored at $-80{ }^{\circ} \mathrm{C}$. Two separate samples of DNA template were provided for NGS $(2.2 \mu \mathrm{g}$ and $4.7 \mu \mathrm{g}$ respectively). In addition, a single walking leg (pereopod) was removed non-destructively from 38 living animals for DNA extraction, for subsequent testing of the developed primers. A $1 / 8$ reaction was prepared for shotgun pyrosequencing on a Roche 454 GS-FLX instrumentation using Titanium chemistry, at the Australian Genome Research Facility (http://www.agrf.com.au). Raw DNA sequence data (46.19 Mbp) was obtained from 184,981 sequence reads, with an average length $250 \mathrm{bp}$. It was calculated that the reported C. cainii data from this study represented approximately $1 \%$ of the average freshwater crayfish genome. Sequences were screened for microsatellites using QDD 2.0 [4], which detected 8799 putative microsatellite loci and PCR primers were designed for 968 microsatellites $(695$ perfect repeats and 273 compound repeats). Loci were selected based on their suitability for multiplex polymerase chain reaction (PCR) and other key parameters (amplicon length between 100 and $350 \mathrm{bp}$, minimum length of flanking region $30 \mathrm{bp}$, maximum number of monobase and di-hexabase repeats in the flanking region of 4 and 2 respectively, and the minimum number of repeats was 5). Optimal primer GC content was set at $50 \%$ (between 40 and $60 \%$ ) and at least one consecutive $\mathrm{G}$ or $\mathrm{C}$ nucleotide was attached to the $3^{\prime}$ end of both the left and right primers (GC clamp). The optimal primer size was set at $20 \mathrm{bp}$ (between 18 and $27 \mathrm{bp}$ ) and the optimal primer melting temperature was set at $60{ }^{\circ} \mathrm{C}$ (between 57 and $63{ }^{\circ} \mathrm{C}$ ). Ten microsatellites were selected for primer design and were tested for PCR amplification on 30 captive C. cainii broodstock. C. quadricarinatus $(n=4)$ and $C$. destructor samples $(n=4)$ were also included for cross-species amplification tests. Prior to PCR, forward primers were tagged with a $5^{\prime}$-M13 universal sequence TGTAAAACGACGGCCAGT [5] and labelled with one of four dyes; FAM, NED, PET or VIC [6]. Genomic DNA (gDNA) was quantified to $50 \mathrm{ng} / \mu \mathrm{L}$ (Nanodrop Technologies ND-1000) for each sample prior to PCR amplification in $10 \mu \mathrm{L}$ reactions, which included $1 \mathrm{X}$ buffer, $0.2 \mathrm{mM}$ dNTPs, $0.1 \mathrm{mg}$ BSA, $0.5 \mathrm{U}$ mango Taq polymerase (Bioline), $1.5 \mathrm{mM} \mathrm{MgCl} 2,0.1 \mu \mathrm{M}$ of forward primer, $0.2 \mu \mathrm{M}$ of reverse primer and fluorescently labelled M13 primer. Initially, PCR amplification was carried out separately for each microsatellite locus to determine optimum
PCR conditions using a $63-55{ }^{\circ} \mathrm{C}$ touchdown program [7]; $94{ }^{\circ} \mathrm{C}$ for $3 \mathrm{~min}$, followed by a 32 cycles touchdown $\left(94{ }^{\circ} \mathrm{C} / 20 \mathrm{~s} ; 63{ }^{\circ} \mathrm{C}\right.$ down to $55{ }^{\circ} \mathrm{C}$ until fifth cycle $/ 45 \mathrm{~s}$; $72{ }^{\circ} \mathrm{C} / 60 \mathrm{~s}$ ), and $72{ }^{\circ} \mathrm{C}$ for $4 \mathrm{~min}$. The PCR products were determined on an ABI 3130 Sequencer (Applied Biosystems) and profiles examined using GENEMAPPER 4.0 (Applied Biosystems) software. Two of the 10 loci failed to amplify for all samples and were subsequently excluded from further analysis. To test for the presence of null alleles, large allele dropout and scoring errors we utilised MICRO-CHECKER 2.2.3 [8], applying $99 \%$ confidence intervals for Monte Carlo simulations. Measures of genetic diversity, such as the number of alleles $(A)$, observed $\left(H_{o}\right)$ and expected $\left(H_{e}\right)$ heterozygosity were estimated with GENALEX 6.5 [9]. The inbreeding coefficient $\left(F_{i s}\right)$ and tests for Hardy-Weinberg Equilibrium (HWE) were estimated in GENEPOP 4.1 [10] and significance was determined with sequential Bonferroni correction [11]. For the calculation of $F_{i s}$ [12], exact $P$-values under the Markov Chain method were implemented with a dememorization step of 10,000, followed by 20 batches (100 batches for LD) of 5000 iterations per batch. To determine relatedness values and the relationships between captive marron individuals, ML-RELATE was utilised [13]. Dyads were found to comprise a parent offspring, full-sib, halfsib or unrelated relationship.

All C. cainii samples amplified at all eight loci and locus Cca10 was monomorphic for 30 samples (Additional file 1: Table S1). The average number of alleles per polymorphic locus was 4.7 (ranging from 2 to 14), and overall average $H_{e}$ was 0.474 . Deviations from HWE were detected at two loci ( $C c a 06$ and $C c a 07)$. However, given that no null alleles were detected, the latter result is probably attributed to the inbred nature of captive samples [14]. Microsatellite markers have previously been described for C. cainii [2], which reported a similar number of alleles (ranging from 2 to 11) and levels of $H_{e}$ (average $H_{e}=0.472$ ). From the cross-species amplification tests, $C$. destructor samples amplified at four of the eight loci and C. quadricarinatus at two loci. Monomorphic loci were detected at $C c a 09$ and $C c a 10$ respectively for each species, however, these results should be reassessed with a larger sample size. Upon investigating the relationships between 30 captive $C$. cainii individuals, $79 \%$ of dyads were assigned as unrelated, $9 \%$ parent offspring, $7 \%$ half-sib and $5 \%$ full-sib. Overall relatedness was estimated at 0.12 , however, no reference population was available for comparison to relatedness values. Due to the ease of scorability and polymorphic nature of the developed microsatellite loci, the markers are suitable for studies of genetic variation in both wild and captive stocks of C. cainii, with potential for cross amplification in other Cherax species. 


\section{Availability of supporting data}

The microsatellite sequences are available through the National Centre for Biotechnology Information (see http://www.ncbi.nlm.nih.gov/). The following accession numbers are available in the GenBank repository; accession no. KP334240 to KP334247. The full list of microsatellites with designed primers $(\mathrm{n}=968)$ is available at LabArchives DOI 10.6070/H4P26W45.

\section{Additional file}

Additional file 1: Table S1. Characterisation of 8 microsatellite loci for 30 Cherax cainii individuals.

\section{Abbreviations}

NGS: next-generation sequencing; PCR: polymerase chain reaction; $A$ : number of alleles; $H_{0}$ : observed heterozygosity; $H_{e}$ : expected heterozygosity; $F_{i s}$ : inbreeding coefficient; HWE: Hardy-Weinberg equilibrium.

\section{Authors' contributions}

SL, LB and NR contributed to the design of the study and drafted the manuscript. SL implemented the study, performed microsatellite marker validation, data analysis and the interpretation of results. All authors read and approved the final manuscript.

\section{Author details}

${ }^{1}$ Molecular Ecology Laboratory, School of Biological Sciences, Flinders University, PO Box 2100, Adelaide 5001, SA, Australia. ${ }^{2}$ Nofima, PO Box 5010, $\mathrm{N}-1432 \AA$ As, Norway.

\begin{abstract}
Acknowledgements
We thank Dean Jerry from the Centre for Sustainable Tropical Fisheries and Aquaculture, James Cook University and John Luckens from Kangabbie Marron Farm for providing samples. Also thanks to Jonathon Sandoval-Castillo from MELFU for laboratory assistance. This study was supported by Flinders University and an AJ \& IM Naylon scholarship, and was registered by the Flinders University Animal Welfare Committee as an invertebrate project conducted in South Australia not requiring ethics approval.
\end{abstract}

\section{Compliance with ethical guidelines}

\section{Competing interests}

The authors declare they have no competing interests.

Received: 27 March 2015 Accepted: 12 August 2015

Published online: 25 August 2015

\section{References}

1. Austin CM, Ryan SG. Allozyme evidence for a new species of freshwater crayfish of the genus Cherax Erichson (Decapoda: Parastacidae) from the south-west of Western Australia. Invertebr Syst. 2002;16:357-67.

2. Kennington JW, Guildea C, Lukehurst SS, Hitchen Y, Gardner MG, Duffy R et al. Isolation and characterization of 13 polymorphic microsatellite loci for the smooth Cherax cainii and hairy marron C. tenuimanus (Decapoda: Parastacidae). Conserv Genet Resour. 2014;6:337-9.

3. Sunnucks P, Hales DF. Numerous transposed sequences of mitochondrial cytochrome oxidase I-II in Aphids of the genus Sitobion (Hemiptera: Aphididae). Mol Biol Evol. 1996:13:510-24.

4. Meglécz E, Costedoat C, Dubut V, Gilles A, Malausa T, Pech N, et al. QDD: a user-friendly program to select microsatellite markers and design primers from large sequencing projects. Bioinformatics. 2010;26:403-4.

5. Schuelke M. An economic method for the fluorescent labelling of PCR fragments. Nat Biotechnol. 2000;18:233-4.

6. Blacket MJ, Robin C, Good RT, Lee SF, Miller AD. Universal primers for fluorescent labelling of PCR fragments-an efficient and costeffective approach to genotyping by fluorescence. Mol Ecol Resour. 2012;12:456-63.

7. Beheregaray LB, Möller LM, Schwartz TS, Chao NL, Caccone G. Microsatellite markers for the cardinal tetra Paracheirodon axelrodi, a commercially important fish from central Amazonia. Mol Ecol Notes. 2004;4:330-2.

8. Van Oosterhout C, Hutchinson WF, Wills DPM, Shipley P. MICRO-CHECKER: software for identifying and correcting genotyping errors in microsatellite data. Mol Ecol Notes. 2004;4:535-8.

9. Peakall R, Smouse PE. GENALEX 6.5: genetic analysis in Excel. Population genetic software for teaching and research—an update. Bioinformatics. 2012;28:2537-9.

10. Rousset F. GENEPOP'007: a complete re-implementation of the genepop software for Windows and Linux. Mol Ecol Resour. 2008:8:103-6.

11. Rice WR. Analyzing tables of statistical tests. Evolution. 1988;43:223-5.

12. Weir BS, Cockerham CC. Estimating F-statistics for the analysis of population structure. Evolution. 1984;38:1358-70.

13. Kalinowski ST, Wagner AP, Taper ML. ML-RELATE: a computer program for maximum likelihood estimation of relatedness and relationship. Mol Ecol Notes. 2006;6:576-9.

14. Hartl DL, Clark AG. Population substructure. In: Hartl DL, Clark AG, editors. Principles of Population Genetics. Sunderland: Sinauer Associates; 1997. p. 111-62.

\section{Submit your next manuscript to BioMed Central and take full advantage of:}

- Convenient online submission

- Thorough peer review

- No space constraints or color figure charges

- Immediate publication on acceptance

- Inclusion in PubMed, CAS, Scopus and Google Scholar

- Research which is freely available for redistribution

Submit your manuscript at www.biomedcentral.com/submit
( Biomed Central 